\title{
The Ascidian Numb Gene Involves in the Formation of Neural Tissues
}

\author{
Hong Ryul Ahn' and Gil Jung Kim ${ }^{\dagger}$ \\ Dept. of Marine Molecular Biotechnology, Gangneung-Wonju National University, Gangneung 210-702, Korea
}

\begin{abstract}
Notch signaling plays fundamental roles in various animal development. It has been suggested that Hr-Notch, a Notch homologue in the ascidian Halocynthia roretzi, is involved in the formation of peripheral neurons by suppressing the neural fates and promoting the epidermal differentiation. However, roles of Notch signaling remain controversial in the formation of nervous system in ascidian embryos. To precisely investigate functions of Notch signaling, we have isolated and characterized Hr-Numb, a Numb homologue which is a negative regulator of Notch signaling, in $H$. roretzi. Maternal expression of $\mathrm{Hr}$-Numb mRNAs was detected in egg cytoplasm and the transcripts were inherited by the animal blastomeres. Its zygotic expression became evident by the early neurula stage and the transcripts were detected in dorsal neural precursor cells. Suppression of Hr-Numb function by an antisense morpholino oligonucleotide resulted in larvae with defect in brain vesicle and palps formation. Similar results have been obtained by overexpression of the constitutively activated $H r-N o t c h$ forms. Therefore, these results suggest that Hr-Numb is involved in Notch signaling during ascidian embryogenesis.
\end{abstract}

Key words : Numb, Neural specification, Notch signaling, Ascidian.

\section{INTRODUCTION}

Notch signaling is used in a variety of cellular contexts during animal development to specify distinct cell fate among individual cells. In vertebrate embryos, Notch signaling is important for the formation of central nervous system (CNS), proliferation of the undifferentiated neural progenitor cells, and generating a variety of neural cell types (Latimer \& Appel, 2006; Artavanis-Tsakonas \& Muskavitch, 2010). In Drosophila, the sensory organ precursor cells develop from a group of equipotent neurogenic epithelial cells, which use Notch signaling to select neural progenitor cells (Campos-Ortega, 1995; Lai, 2004). The neural progenitor cells again use Notch signaling to specify different cell fates following asymmetric cell division. The asymmetric cell

\footnotetext{
${ }^{\dagger}$ Corresponding author: Gil Jung Kim, Dept. of Marine Molecular Biotechnology, Gangneung-Wonju National University, 7 Jukheon-gil, Gangneung 210-702, Korea. Phone: +82-33-640-2415, Fax: +82-33-640-2849, E-mail: gjkim@gwnu.ac.kr

${ }^{1}$ Present address: Functional Food Center, Korea Institute of Science and Technology, Gangneung Institute, Gangneung 210-340, Korea
}

division gives rise to a pIIa cell in which the Notch signaling becomes activated (non-neural fate) and a pIlb cell in which this signaling remains inactive (neural fate). The difference in Notch signaling activity is established by Numb (Uemura et al., 1989; Guo et al., 1996). In this process, Numb protein is asymmetrically segregated and localized to the pIIb daughter cell, in which suppresses Notch signaling activity. Numb encodes an adaptor protein that is able to bind the Notch receptor and interacts with endocytic proteins such as alpha-adaptin and EPS15, and then functions to promote the pIIb cell fate (Santolini et al., 2000; Berdnik et al., 2002). Recently, it was reported that Numb inactivates Notch by promoting its endocytosis during asymmetric cell division in Drosophila embryos (Couturier et al., 2012). Interaction of Numb with the Notch signaling pathway appears to be conserved in animal development, particularly in specification of the neural precursor cells (Kandachar \& Roegiers 2012).

Ascidians belong to the Urochordata. They are one member of the phylum Chordata with vertebrates and cephalochordates. The ascidian tadpole larvae possess a 
typical chordate body plan with a CNS composed of only $\sim 100$ neurons (Meinertzhagen et al., 2004). The simplicity of ascidian CNS makes it an ideal model for understanding the development and function of chordate-specific neuronal networks. Notch signaling is also involved in specification of the neural progenitor cells and neural tube patterning in ascidian embryos. Overexpression of Notch led to defects in neural tube closure and in the formation of brain vesicle, palps, and peripheral neurons (Akanuma et al., 2002). It was also reported that a Delta2/Notchmediated relay from the posterior motor ganglion specifies the fate of anterior motor ganglion (Stolfi et al., 2011). During ascidian embryogenesis, however, function of Numb in Notch signaling remains unknown.

In this study, we isolated and characterized the ascidian Numb gene. The ascidian Numb transcript was found to be expressed both maternally and zygotically. Its zygotic expression is observed in dorsal neural precursor cells from the early neural stage embryo. Larvae injected with Numb morpholino oligonucleotide showed abnormalities in brain and palps formation, but not pigment cells.

\section{MATERIALS AND METHODS}

\section{Animals and embryos}

Adults of the ascidian Halocynthia roretzi were collected or purchased from fishermen in the vicinity of the Marine Biology Center for Research and Education at GangneungWonju National University, Gangneung, Korea. Naturally spawned eggs were fertilized with a suspension of sperm from another individual, and then raised in filtered seawater containing $50 \mu \mathrm{g} / \mathrm{mL}$ streptomycin sulfate and $50 \mu \mathrm{g} / \mathrm{mL}$ kanamycin sulfate at $10-13{ }^{\circ} \mathrm{C}$. Embryos were collected at appropriate stages and fixed for whole-mount in situ hybridization.

2. Isolation and characterization of cDNA clone for Halocynthia Numb gene

The cDNA fragments encoding a part of N-terminus region of Halocynthia Numb were isolated by RT-PCR with degenerate oligonucleotides, 5'-CA(A/G)TGGCA(A/G) $(\mathrm{A} / \mathrm{C} / \mathrm{G})(\mathrm{C} / \mathrm{A}) \mathrm{IGA}(\mathrm{C} / \mathrm{T}) \mathrm{GA}(\mathrm{A} / \mathrm{G}) \mathrm{G}-3$ ' for the upstream, 5'-(G/A)AAIGC(G/A)CAICCIACIGC(G/A)T-3' for the downstream, and 5'-(A/G)GTI(A/T)(G/C)ITT(C/T)TG(C/T) GCICCIGA-3' for the nested downstream primers from gastrula stage poly(A) RNA. Larger fragments of $\mathrm{Hr}-\mathrm{Numb}$ covering the complete ORF were obtained by 5' and 3' rapid amplification of cDNA ends (RACE) using a SMART RACE cDNA amplification kit (Clontech). The primers for RACE were the following: 5'-GTCGTGATGG AACTACGAGACGCTGGATATG-3' for the upstream and 5'-GGCTTAAACGTTCACCCGAATCCTTAATGGC3 ' for the downstream. Molecular phylogenetic relationships among the Numb products were estimated with MEGA 5.05 program using the neighbor-joining method (Saitou $\&$ Nei, 1987). Sequence data used in this study were taken from GenBank databases, with following accession numbers: Homo a, Homo sapiens Numb isoform CRA-a (EAW81100.1); Homo d, Homo sapiens Numb isoform CRA-d (EAW81110.1); Homo e, Homo sapiens Numb isoform CRA-e (EAW81104.1); Homo f, Homo sapiens Numb isoform CRA-f (EAW81114.1); Mus 1, Mus musculus Numb1 (AAD47835.1); Mus 2, Mus musculus Numb2 (AAD47836.1); Gallus, Gallus gallus Numb (AAD49434.1); Xenopus, Xenopus tropicalis Numb (CAL49325.1); Danio 1, Danio rerio Numb (AAT85677.1); Danio 2, Danio rerio Numb-like (AAI07954.1); Ciona, Ciona intestinalis Numb (BAE06599.1); Drosophila, Drosophila melanogaster Numb isoform A (AAF52776.1).

\section{Whole-mount in situ Hybridization}

Whole-mount in situ hybridization was performed using a digoxigenin-labeled $\mathrm{Hr}$-Numb antisense probe, as described previously (Miya et al., 1997; Lee et al., 2011). Specimens were hybridized with the probe at $50^{\circ} \mathrm{C}$.

\section{Microinjection of MOs}

To suppress functions of Hr-Numb, we injected the 
morpholino antisense oligonucleotide ( $\mathrm{MO}$, Gene Tools) into eggs as described by Kim et al. (2007). The nucleotide sequence of $H r$-Numb MO was 5'-GCTTTGTCTTAT TGTCCTTATCATG-3'. The standard control MO provided by Gene Tools was used as a control experiment. MOs were dissolved in sterile distilled water with Fast Green and injected into fertilized eggs. The final concentration of each MO to be injected was approximately $1 \mathrm{mg} / \mathrm{mL}$. The injected eggs were allowed to develop up to hatching stage.

\section{RESULTS AND DISCUSSION}

To clarify the mechanism by which Notch signaling specify neural cells during ascidian embryogenesis, we isolated Numb homologue (Hr-Numb) in the ascidian Halocynthia roretzi by the classical RT-PCR and 5' and 3' RACE. In various Numb proteins, the N-terminal region, which contains the phosphotyrosine-binding (PTB) domain interacting with a wide variety of different proteins, is highly conserved among vertebrates and invertebrates (Berdnik et al., 2002; Reugels et al., 2006). Using degenerate oligonucleotide primers (see Materials and Methods), we amplified target fragments from the Halocynthia gastrula stage poly(A) RNA by RT-PCR. Then, a fragment of Halocynthia Numb covering the complete ORF was obtained by RACE. A cDNA clone for $H r-N u m b$ was 1,624 base pairs in full length, and the predicted protein has 456 amino acids (Fig. 1). The predicted amino acid sequence contained a well-conserved $\mathrm{N}$-teminus sequence between various Numb homologues. Although the over degree of amino acid identity was not high (about $30 \%$ ), the amino acid sequence of Hr-Numb PTB domain was highly conserved when compared with the PTB domains of other Numb proteins (data not shown).

In order to understand the relationships among the Numb genes from various animal groups, we assembled a molecular phylogenetic tree (Fig. 2). The phylogenetic tree clearly shows that Hr-Numb is an Halocynthia orthologue of vertebrate Numb. Numb-like gene (e.g. Danio 2 in Fig. 2) is a related member of the Numb family of endocytosis promoting genes (Niikura et al., 2006). Numb and Numblike share high sequence similarities at amino acid level. However, Numb-like has two specific motifs that were not found in Numb, in the N-terminal and middle regions (Zhong et al., 1997; Niikura et al., 2006). Hr-Numb did not contain these motifs, suggesting to be an orthologue of Numb rather than that of Numb-like. There is no report that Ciona intestinalis, another ascidian species, has a Numb-like gene.

Whole-mount in situ hybridization demonstrated that the maternal transcripts of $\mathrm{Hr}-\mathrm{Numb}$ are distributed evenly in the egg cytoplasm (Fig. 3A). Cleavage of Halocynthia eggs is bilaterally symmetrical. At the 8-cell stage, the transcripts were distributed in the cytoplasm of the animal pole-side blastomeres, although the signal was weak in the vegetal pole-side blastomeres (Fig. 3B, b). This expression pattern was inherited by the early gastrula stage. The maternal transcripts became concentrated near the cytoplasm of the animal blastomeres (Fig. 3c-f). Zygotic expression of $\mathrm{Hr}$-Numb was firstly observed in the dorsal neural precursor cells at the early neurula stage (Fig. 3G, arrows). At the tailbud stage, the expression became strongly in the A- and a-lineage neural precursor cells of the dorsomedial head region (Fig. $3 \mathrm{H})$. These cells give rise to brain, peripheral neurons, and palps. The expression pattern of $H r-N u m b$ is similar to that of Hr-Notch reported by Hori et al. (1997). $\mathrm{Hr}$-Notch was maternally expressed and its zygotic expression was visible in the dorsal part of the A-lineage nerve cord precursor, and in the a-line brain and palps precursor cells at the neurula stage. Thus, it is suggested that Hr-Numb is involved in Notch signaling in which specify the anterior region of neural precursor cells during ascidian embryogenesis. The expression pattern of $\mathrm{Hr}-\mathrm{Numb}$ is also similar to that of zebrafish Numb. In zebrafish, Numb transcripts were detectable in all regions of the embryos at the mid-blastula transition 


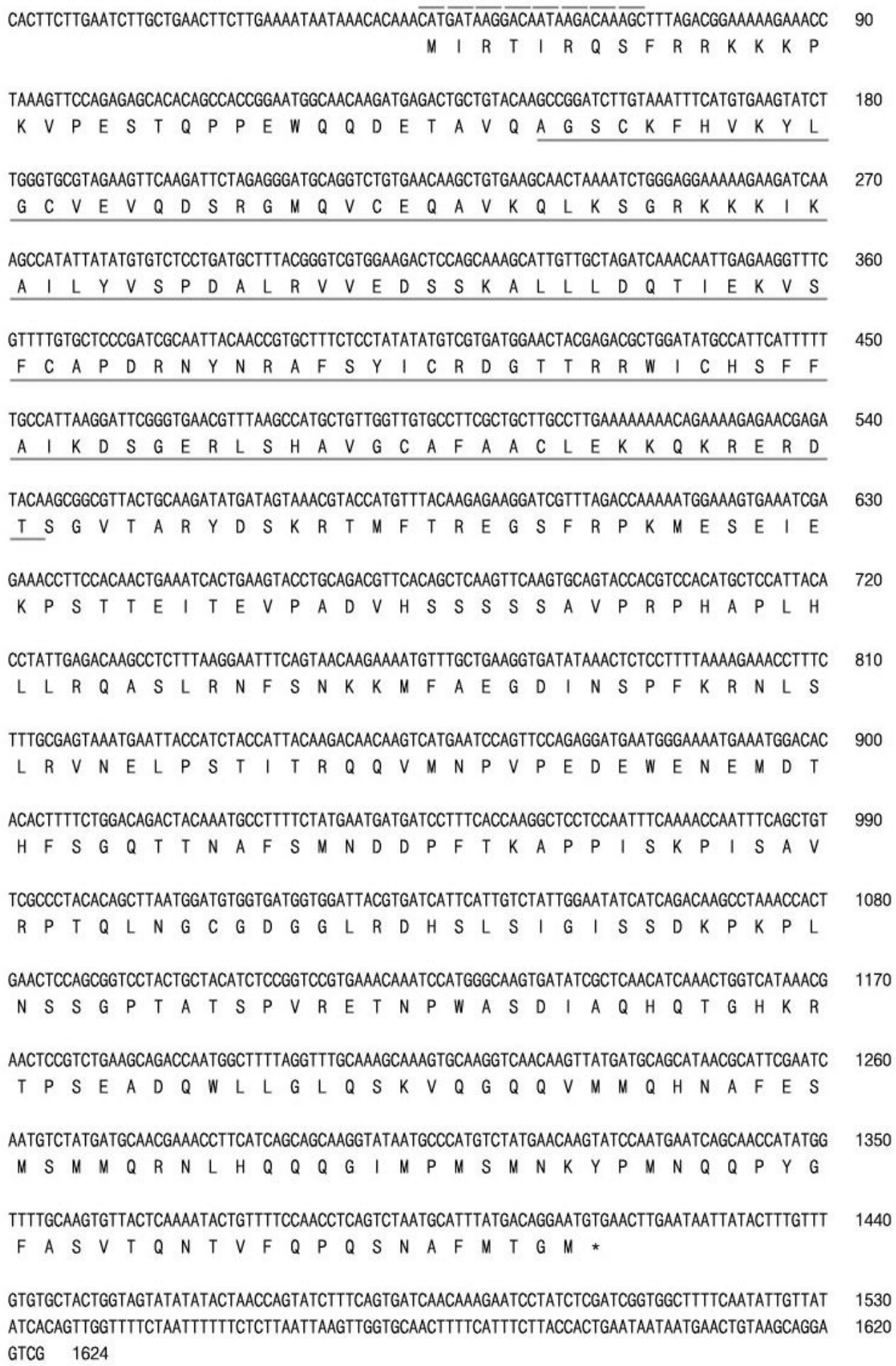

Fig. 1. The nucleotide and deduced amino acid sequences of a cDNA clone for the $\boldsymbol{H r}$ - $\mathbf{N u m b}$. The sequence of the cDNA encompasses 1,624 bp including 5' and 3'untranslational regions. The ATG at the position 47-49 represents the putative start codon of the $H r$-Numb-encoded protein of 456 amino acids. The termination codon is shown by an asterisk and the PTB domain is underlined. The 25 base pairs for MO design are shown by dotted lines around the putative start codon. 


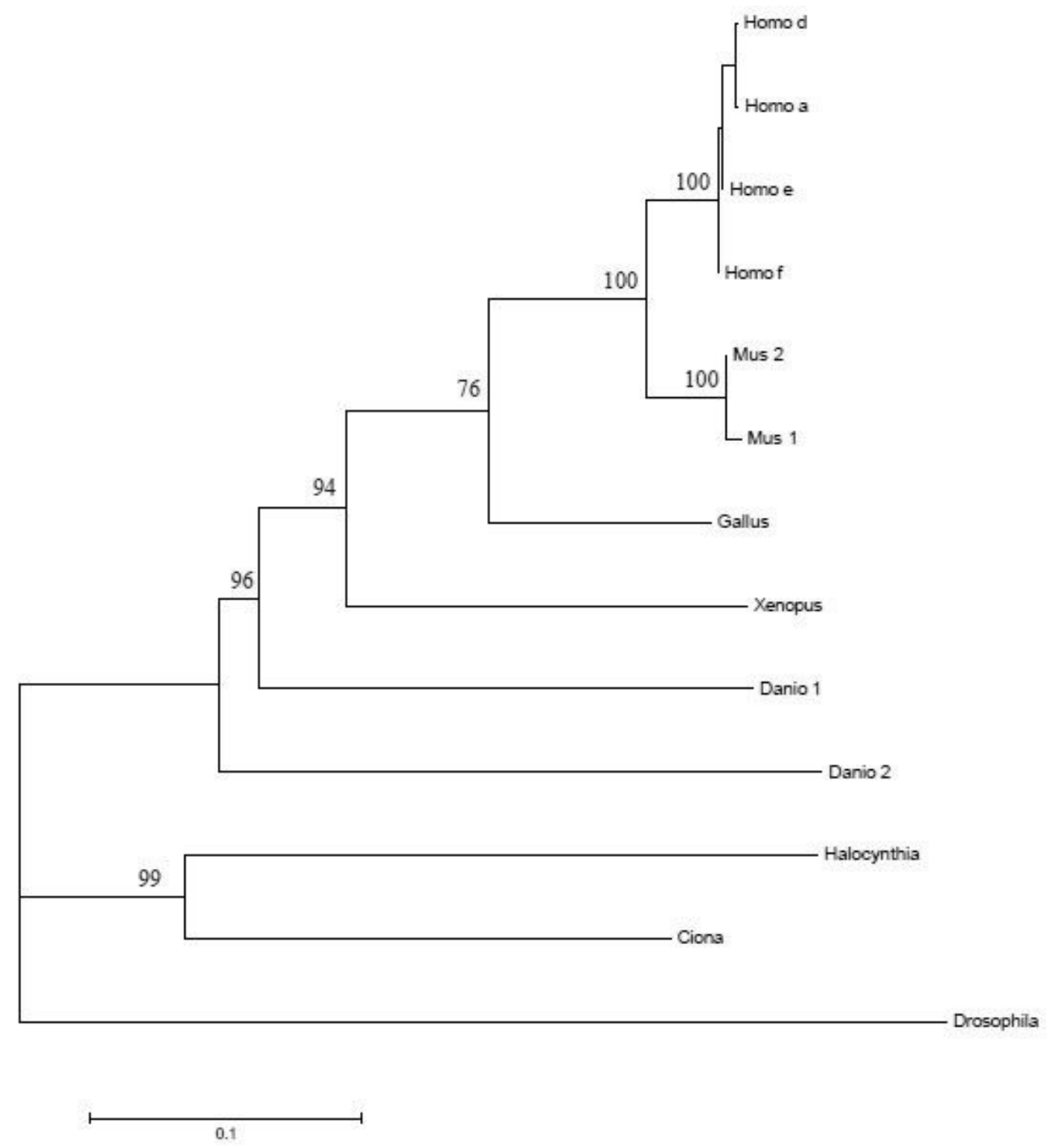

Fig. 2. Molecular phylogenetic tree of Numb family members. Phylogenetic relationships for amino acid sequences of the Numb family members including Halocynthia inferred from the neighbor-joining analysis with Drosophila sequence as outgroup. Results from bootstrap resampling (1,000 replicates) are shown above internal nodes. The scale bar indicates an evolutionary distance of 0.1 amino acid substitutions per sites.

stage (Reugels et al., 2006). Expression of zebrafish Numb was found in the midline from the head to the tail regions at the neurula stage, and then the signal was restricted in the brain and eye progenitor cells at the later stage. Therefore, it is likely that roles of Numb protein are conserved in Notch signaling, which specify neural precursor cells in chordate development.

Next, we attempted to inhibit functions of Hr-Numb by injecting fertilized eggs with antisense morpholino oligonucleotide (MO). Antisense MO prevents translation of the coding region of the targeted mRNA. The timing of initiation of gastrulation was delayed about one hour in the Hr-Numb MO injected embryos $(85 \%, n=47)$ compared with normal embryos. Injection of $\mathrm{Hr}$-Numb MO resulted in the loss of brain vesicle (Fig. 4B, black arrow) and palps (Fig. 4B, red arrow) in $89 \%(n=47)$ 

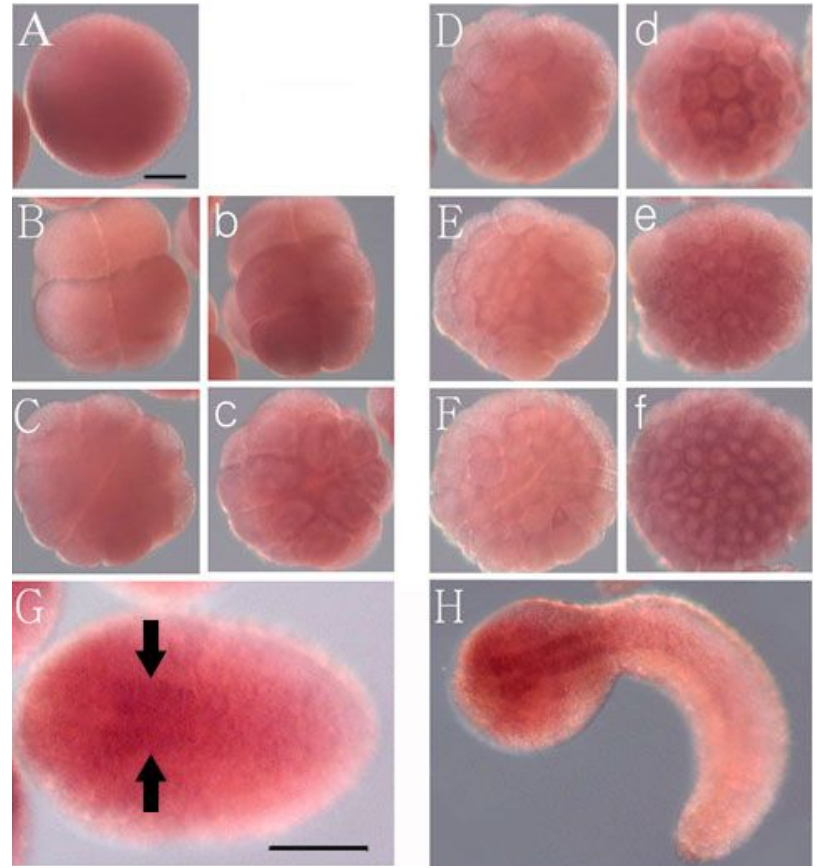

Fig. 3. Whole-mount in situ hybridization pattern of $\mathbf{H r}$-Numb expression during Halocynthia embryogenesis. (A) A fertilized egg. (B, b) 8-, (C, c) 16-, (D, d) 32-, (E, e) 64-, (F, f) 110-cell stage embryos. (B-F) Vegetal pole views. (b-f) Animal pole views. (G) An early neurula. Anterior is to the left. Arrows indicate zygotic expression of $H r-N u m b$ in the dorsoanterior neural precursor cells. (H) A tailbud stage embryo. Hr-Numb is expressed in the dorsal neural cells. Scale bars $=100 \mu \mathrm{m}$.

of cases. However, the Hr-Numb MO-injected larvae showed formation of pigment cells, although the cells located outside of head region (Fig. 4B, black arrow). This might be caused by incomplete neural tube closure. No specific abnormality was observed in the larvae injected with control MO in $91 \%$ of cases $(n=32)$ (Fig. 4A). These results suggest that Hr-Numb is essential for brain vesicle and palps formation, but not for pigment cells formation. Similar results have been reported by Akanuma et al. (2002). Overexpression of the constitutively activated $\mathrm{Hr}$-Notch forms resulted in larvae with defects in neural tube closure and brain vesicle formation. The activated Hr-Notch also suppressed formation of palps, but not pigment cells. Therefore, it is plausible that

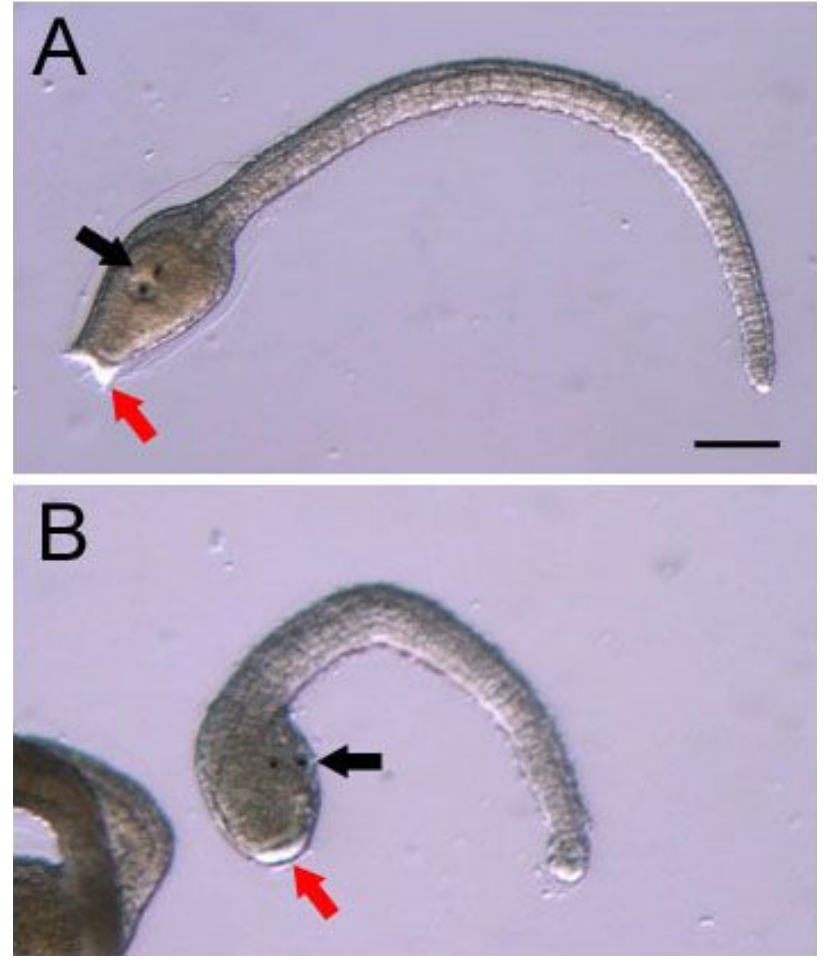

Fig. 4. Effects of $\boldsymbol{H r}$-Numb MO injected into eggs. (A) A larva injected with control MO shows normal morphology. A brain vesicle, pigment cells (black arrow) and palps (red arrow) were formed. Anterior is to the left. (B) A larva injected with $H r-N u m b$ MO. Two pigment cells (black arrow) locate laterally in the outer region of head. Red arrow in B indicates no palps formation. Scale bar $=100 \mu \mathrm{m}$.

Hr-Numb is involved in control of Notch signaling during ascidian embryogenesis.

In zebrafish, Numb and Numb-like are involved in differentiation of primitive erythrocytes (Bresciani et al., 2010). The zebrafish Numb and Numb-like knockdown experiments showed severe reduction or absence of embryonic erythrocytes. There is a report that Notch signaling controls the Numb protein level. In the developing chick CNS, a reciprocal negative regulation presents between Notch and Numb proteins, namely, high levels of Notch activation cause a reduction in the Numb and Numb-like protein levels (Chapman et al., 2006). Inhibition of Notch signaling by Numb is essential for various cell fate decisions. Future studies using Hr-Numb may 
provide new insights for Notch signaling during ascidian embryogenesis.

\section{ACKNOWLEDGEMENT}

This work was supported by a Gangneung-Wonju National University Research Grant (2010-0150) to G.J.K.

\section{REFERENCES}

Akanuma T, Hori S, Darras S, Nishida H (2002) Notch signaling is involved in nervous system formation in ascidian embryos. Dev Genes Evol 212:459-472.

Artavanis-Tsakonas S, Muskavitch MA (2010) Notch: the past, the present, and the future. Curr Top Dev Biol 92:1-29.

Berdnik D, Török T, González-Gaitán M, Knoblich JA (2002) The endocytic protein alpha-Adaptin is required for numb-mediated asymmetric cell division in Drosophila. Dev Cell 3:221-231.

Bresciani E, Confalonieri S, Cermenati S, Cimbro S, Foglia E, Beltrame M, Di Fiore PP, Cotelli F (2010) Zebrafish numb and numblike are involved in primitive erythrocyte differentiation. PLoS One 5:e14296.

Campos-Ortega JA (1995) Genetic mechanisms of early neurogenesis in Drosophila melanogaster. Mol Neurobiol 10:75-89.

Chapman G, Liu L, Sahlgren C, Dahlqvist C, Lendahl U (2006) High levels of Notch signaling down-regulate Numb and Numblike. J Cell Biol 175:535-540.

Couturier L, Vodovar N, Schweisguth F (2012) Endocytosis by Numb breaks Notch symmetry at cytokinesis. Nat Cell Biol 14:131-139.

Guo M, Jan LY, Jan YN (1996) Control of daughter cell fates during asymmetric division: interaction of Numb and Notch. Neuron 17:27-41.

Hori S, Saitoh T, Matsumoto M, Makabe KW, Nishida H (1997) Notch homologue from Halocynthia roretzi is preferentially expressed in the central nervous system during ascidian embryogenesis. Dev Genes Evol 207:371-380.

Kandachar V, Roegiers F (2012) Endocytosis and control of Notch signaling. Curr Opin Cell Biol 24:534-540.

Kim GJ, Kumano G, Nishida H (2007) Cell fate polarization in ascidian mesenchyme/muscle precursors by directed FGF signaling and role for an additional ectodermal FGF antagonizing signal in notochord/ nerve cord precursors. Development 134:1509-1518.

Lai EC (2004) Notch signaling: control of cell communication and cell fate. Development 131:965-973.

Latimer AJ, Appel B (2006) Notch signaling regulates midline cell specification and proliferation in zebrafish. Dev Biol 298:392-402.

Lee WY, Ham HS, Kim GJ (2011) Expression of Wee1 gene in the ascidian, Halocynthia roretzi embryo. Dev Reprod 15:1-7.

Meinertzhagen IA, Lemaire P, Okamura Y (2004) The neurobiology of the ascidian tadpole larva: recent developments in an ancient chordate. Annu Rev Neurosci 27:453-485.

Miya T, Morita K, Suzuki A, Ueno N, Satoh N (1997) Functional analysis of an ascidian homologue of vertebrate Bmp-2/Bmp-4 suggests its role in the inhibition of neural fate specification. Development 124:51495159 .

Niikura Y, Tabata Y, Tajima A, Inoue I, Arai K, Watanabe S (2006) Zebrafish Numb homologue: phylogenetic evolution and involvement in regulation of left-right asymmetry. Mech Dev 123:407-414.

Reugels AM, Boggetti B, Scheer N, Campos-Ortega JA (2006) Asymmetric localization of Numb: EGFP in dividing neuroepithelial cells during neurulation in Danio rerio. Dev Dyn 235:934-948.

Saitou N, Nei M (1987) The neighbor-joining method: a new method for reconstructing phylogenetic trees. Mol Biol Evol 4:406-425.

Santolini E, Puri C, Salcini AE, Gagliani MC, Pelicci PG, Tacchetti C, Di Fiore PP (2000) Numb is an 
endocytic protein. J Cell Biol 151:1345-1352.

Stolfi A, Wagner E, Taliaferro JM, Chou S, Levine M (2011) Neural tube patterning by Ephrin, FGF and Notch signaling relays. Development 138:5429-5439. Uemura T, Shepherd S, Ackerman L, Jan LY, Jan YN (1989) Numb, a gene required in determination of cell fate during sensory organ formation in Drosophila embryos. Cell 58:349-360.
Zhong W, Jiang MM, Weinmaster G, Jan LY, Jan YN (1997) Differential expression of mammalian Numb, Numblike and Notch1 suggests distinct roles during mouse cortical neurogenesis. Development 124:18871897.

(Received 2 October 2012, Received in revised form 19 October 2012, Accepted 17 November 2012) 